\title{
Optical spectroscopy investigation of peptides issued from the AML1-ETO-E-protein complex relevant to acute myeloid leukemia
}

\author{
H. Porumb * \\ Université Paris 13, 93017 Bobigny Cedex, and LBPA, UMR CNRS 8113, ENS de Cachan, \\ 94235 Cachan, France
}

\begin{abstract}
The expression of AML1-ETO, resulting from the $t(8 ; 21)$ chromosomal translocation causes $15 \%$ of acute myeloid leukaemias. The NHR2 region of ETO, bearing the motif LxxLL, is involved in the oligomerisation of the AML1-ETO. "Peptide NHR2" is one of the objects of the present investigation. The TAFH region of ETO may recruit AML1-ETO to transcription activators, such as E-protein. "Peptide TAFH" is another object of the present investigation. TAFH interacts with E-protein through the AD1 domain of the latter, which possesses an LxxLL motif as well. "Peptide AD1" is the third object of the present investigation. By CD, ANS fluorescence and intrinsic fluorescence, we suggest an antiparallel coiled-coil encounter of two NHR2 molecules $\left(K_{\mathrm{d}}=2.8-4 \mu \mathrm{M}\right)$ as a prerequisite to tetramer formation. On the other hand, we show that the TAFH domain would probably recognize another partner bearing the LxxLL motif and, before binding to AD1 $\left(K_{\mathrm{d}}=28 \mathrm{nM}\right)$, the first such interaction is likely to be intramolecular, with the NHR2 domain of the AML1-ETO protein itself $\left(K_{\mathrm{d}}=1.28 \mathrm{nM}\right)$. Furthermore, a possible interaction of NHR2 with AD1 is also revealed $\left(K_{\mathrm{d}}=240 \mathrm{nM}\right)$. The biological implications of the results are discussed.

Keywords: Activity modulation, amphipathic peptides, ANS, binding constants, circular dichroism, coiled coil, intrinsic fluorescence, Newton-Raphson method, oligomerisation
\end{abstract}

\section{Abbreviations}

ANS, 1-anilinonaphtalene-8-sulfonic acid;

$\mathrm{CD}$, circular dichroism;

$\mathrm{CBF}$, core binding factor;

DMSO, dimethyl sulfoxide;

ETO, 'eight-twenty-one' protein;

$K_{\mathrm{d}}$, dissociation constant;

NHR1, (Drosophila) nervy-homology region 1 domain;

TAFH, TATA-binding protein (TBP)-associated factor (TAF) homology.

\footnotetext{
*Address for correspondence: H. Porumb, PhD, Université Paris 13, 74 rue Marcel Cachin, 93017 Bobigny Cedex, and LBPA, UMR CNRS 8113, ENS de Cachan, 61 Avenue du Président Wilson, 94235 Cachan, France. E-mail: hporumb@hotmail.com.
} 


\section{Introduction}

The molecular characterization of leukaemia has demonstrated that genetic alterations in the leukaemic clone frequently fall into 2 classes, those affecting transcription factors (such as AML1-ETO) and mutations affecting genes involved in signal transduction (such as activating mutations of FLT3 and KIT) [9]. This finding has favoured a model of leukaemogenesis in which the collaboration of these 2 classes of genetic alterations is necessary for the malignant transformation of haematopoietic progenitor cells [10].

LxxLL motifs participate in many protein-protein interactions associated with different aspects of regulation including activation or repression of transcription. Several reported 3D structures of proteinLxxLL motif complexes and an interaction implicated in leukaemia have further highlighted the diversity and regulatory importance of this apparently simple motif [6].

Normally, CBF, the core binding factor, binds, as a dimer, DNA through its AML1 subunit and recruits other transcription factors. The abnormal expression of an AML1-ETO fusion protein, resulting from the $t(8 ; 21)$ chromosomal translocation is responsible for up to $15 \%$ of acute myeloid leukaemias. The function of wild type "eight-twenty-one", or ETO protein is unknown. One possibility in leukaemia is that, having been anchored onto the CBF target DNA via the AML1 subunit, AML1-ETO recruits corepressors and histone deacetylases involved in chromatin repression. The formation of a corepressor complex involving AML1-ETO thus serves to shut down expression of genes normally activated by CBF (Fig. 1(a), adapted after [6]).

We know that a region of ETO (called NHR2) is involved in the oligomerisation of the AML1-ETO protein. It bears the motif LxxLL [6]. "Peptide NHR2" is one of the objects of the present investigation.

Another possible deregulation leading to leukaemia involves a region of ETO called TAFH, from TATA-binding protein (TBP)-associated factor (TAF) homology, also called (Drosophila) nervyhomology region 1 (NHR1) domain, of 104 amino acids. "Peptide TAFH" is another object of the present investigation.

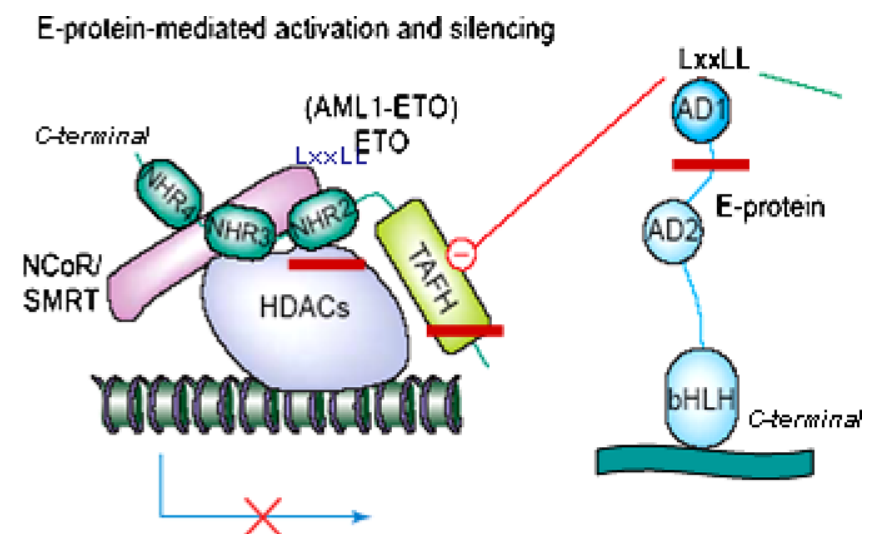

(a)

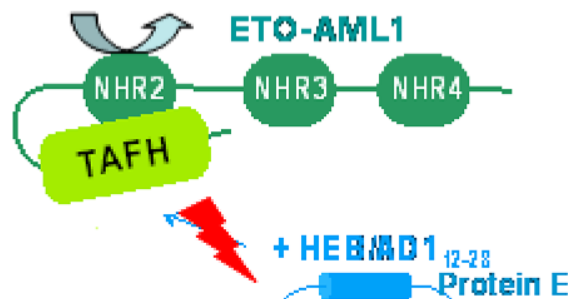

(b)

Fig. 1. Peptides issued from the AML1-ETO-E-protein complex (redrawn after Plevin et al. 2005 [6]). NHR4 is the AML1 domain of CBF, expressed alongside with ETO following the $t(8 ; 21)$ chromosomal translocation. The peptides under investigation are underlined. Note that both NHR2 and AD1 peptides contain LxxLL motifs (a). Hypothetical intramolecular interaction between the NHR2 and TAFH domains of AML1-ETO, which may modulate the oligomerisation of ETO via the NHR2 domain (curved arrow) and/or the interaction with the AML1 subunit of E-protein (flash) (b). 
TAFH region may serve to recruit ETO (or AML1-ETO) to other transcription activators, such as E-protein. E-proteins are a family of transcription factors involved in regulating cell growth, differentiation, and apoptosis. Normally, DNA-bound E-proteins interact with the histone acetyltransferase p300/CREB binding protein, leading to the formation of a complex causing histone modifications that facilitate transcription initiation. AML1-ETO inhibits E-protein-mediated transactivation of transcription by physically displacing wild type coactivator proteins that normally bind to E-protein. AML1-ETO, via its TAFH region, interacts with E-protein through its $\mathrm{N}$-terminal activation domain (AD1). AD1 region (or HEB) contains 99 amino acids incorporating an LxxLL motif as well [6,7]. The 3D solution (NMR) structure of the AD1-bound state of the human ETO-TAFH domain (eTAFH) is known, but the molecular details concerning the bound AD1 segment could not be determined [7]. A helical conformation of AD1 was inferred from sequence conservation and secondary structure predictions based on analogies [3,7]. "Peptide AD1" representing a 17 amino acids fragment of AD1 (positions 12-28) is the next object of the present investigation.

In this work, we looked by optical spectroscopic techniques (CD, ANS fluorescence and intrinsic fluorescence) at peptides NHR2 and TAFH, issued from ETO, and peptide AD1, issued from E-protein respectively. We describe the solution properties and associations of peptide NHR2 and then investigate the mutual interaction taking place among NHR2, TAFH and AD1. A discussion of possible biological implications of the results follows.

\section{Materials and methods}

The peptides used in this work are described in Table 1. Note that peptide NHR2 contains a Trp residue, where as peptide TAFH contains a Tyr.

\subsection{ANS test}

The use of ANS to monitor protein oligomerisation is a standard technique that has been adapted by us to study the association of peptides [8]. The "working buffer" was $15 \mathrm{mM}$ Tris $\mathrm{pH} 7.5,150 \mathrm{mM} \mathrm{NaCl}$. The NHR2 peptide was initially dissolved in water at $2 \mathrm{mM}$ by weight. Subsequent dilutions were made in the working buffer and their concentrations were determined spectrophotometrically. The ANS was

Table 1

Peptides used in this work

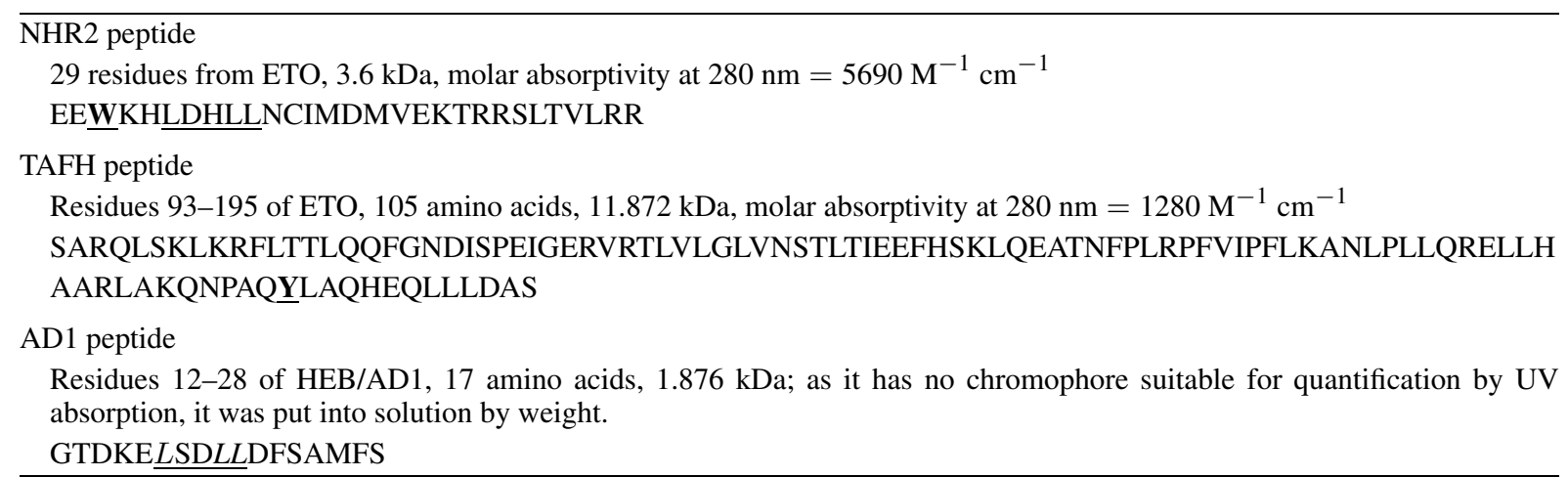

The LxxLL motifs are underlined. Tryptophane and tyrosine residues are highlighted. 
initially dissolved at $5 \mathrm{mM}$ in $10 \% \mathrm{DMSO} /$ water by weight and brought to the desired concentration by subsequent dilution with buffer.

Fluorescence emission spectra of ANS were recorded with a Shimadzu RF-5301 spectrofluorophotometer. To a $1 \mathrm{ml}$ solution of ANS at $50 \mu \mathrm{M}, 0.5 \mu \mathrm{l}$ aliquots of peptide solution of an appropriate concentration were added to cover the concentration range of 0.1-35 $\mu \mathrm{M}$. Fluorescence was excited at $372 \mathrm{~nm}$. Emission spectra were recorded in the range 450-660 nm, with $3 \mathrm{~nm}$ and $5 \mathrm{~nm}$ excitation and emission monochromator slits respectively and with the photo multiplier in the high gain mode.

During the titration by NHR2, the emission wavelength shifted from 542 to $532 \mathrm{~nm}$. The value of the fluorescence quantum yield enhancement, $Q$, was calculated at each peptide concentration, where $Q=F / F_{0}-1, F$ being the ANS fluorescence at the maximum emission wavelength and $F_{0}$ being the maximum fluorescence obtained in the absence of peptide. A plot of $1 / Q$ vs. $1 /$ peptide concentration was made. This plot showed two linear portions that changed slope at a particular breakpoint. On each side of the breakpoint of the graph linear regressions were performed in order to obtain the "maximum fluorescence enhancement at infinite peptide concentration" within those protein concentration ranges. The change in slope is assumed to occur at the concentration that measures the middle of the peptide association process.

\subsection{Circular dichroism}

\subsubsection{NHR2 peptide association}

Circular dichroism spectra were recorded with an AVIV 60DS V4.1t dichrograph in a $0.5 \mathrm{~cm}$ quartz cell at $25^{\circ} \mathrm{C}$, in the wavelength range $200-260 \mathrm{~nm}$, at $0.5 \mathrm{~nm}$ intervals, with $1 \mathrm{~nm}$ bandwidth and $8 \mathrm{~s}$ integration time. The peptide concentrations were varied by successive dilutions from 18 to $1.125 \mu \mathrm{M}$ in working buffer. The absolute values of the molar differential absorptivities per residue at $222 \mathrm{~nm}$ were plotted against peptide concentration.

\subsection{Intrinsic fluorescence titrations}

\subsubsection{Stoichiometry of the interaction of peptides NHR2 and TAFH}

Fluorescence emission spectra from peptide NHR2, containing a Trp residue, were recorded with the Shimadzu RF-5301 spectrofluorophotometer in a semi-micro cell ( $800 \mu \mathrm{l}$ volume) at $25^{\circ} \mathrm{C}$, in the 300 $500 \mathrm{~nm}$ wavelength range, with excitation at $295 \mathrm{~nm}$, using $3 \mathrm{~nm}$ excitation and emission monochromator slits and the photo multiplier in the high gain mode. The TAFH peptide was diluted to $50 \mu \mathrm{M}$ in the working buffer. The cell contained NHR2 peptide at 0.5 or $1.0 \mu \mathrm{M}$ concentration and aliquots from the $50 \mu \mathrm{M}$ TAFH peptide solution were added to cover the range up to $2.5 \mu \mathrm{M}$. The fluorescence intensities at the maximum emission wavelength (at about $346 \mathrm{~nm}$ ) were obtained from the baseline-corrected spectra and, after taking into account the dilution effect, were plotted against protein concentration in order to obtain the binding stoichiometry.

\subsubsection{Dissociation constants for the interaction of TAFH with peptides NHR2 and AD1}

The binding constants for the interaction of peptide TAFH with peptides NHR2 and AD1 were determined by adapting a program obtained from, and described by Linse et al. [1,2]. The procedure consists in an iterative least squares fit directly to the measured data i.e., to the fluorescence of NHR2 peptide at $346 \mathrm{~nm}$, maintained at approximately constant concentration (with dilution taken into account), as a function of total added peptide concentration in the absence, or in the presence, of a known concentration of a third peptide, considered to be a competitor. Thus the Trp fluorescence from NHR2 was 
monitored as a function of TAFH concentration, in the absence of any competitor, for the TAFH-NHR2 interaction; as a function of TAFH in the presence of AD1 as competitor, for the binding of TAFH to $\mathrm{AD} 1$; and as a function of AD1 alone, for the mutual interaction of AD1 and NHR2. The titration data of NHR2 by TAFH were first corrected for the contribution of the tyrosine present in TAFH by subtracting the TAFH intensities, recorded separately. For each set of values of the variables, the Newton-Raphson method was then used to solve for the free protein concentration at each titration point, followed by the determination of the (macroscopic) binding constants of each of the interactions. The uncertainties in the $K_{\mathrm{d}}$ values are thought to be of the order of $10 \%$. It should be noted that the results are critically dependent on the accurate knowledge of the concentrations of the partners.

\section{Results and discussion}

\subsection{NHR2 peptide association}

By the ANS test, the presence of a well-defined breakpoint in the $1 / Q$ vs. $1 /$ peptide concentration plot suggests that the peptide oligomerisation process is highly cooperative (Fig. 2). Oligomerisation is onset at about $4.2 \mu \mathrm{M}$ concentration. This figure is consistent with the value of $5.7 \mu \mathrm{M}$ (obtained as the third order root of $190 \mu \mathrm{M}^{3}$ ), suggested by analytical ultra centrifugation. Indeed, ultra centrifugation (inset to Fig. 2) suggests that NHR2 exists in a monomer - tetramer equilibrium with $K_{\mathrm{d}}=190 \mu \mathrm{M}^{3}$. This is particularly reassuring, given that a tetramer structure of the NHR2 domain is known to be critical for the AML1-ETO activity [3].

The low value $(Q=0.56)$ of the fluorescence enhancement at infinite peptide concentration in the low peptide concentrations range suggests that the species is probably a monomer below $4.2 \mu \mathrm{M}$. This

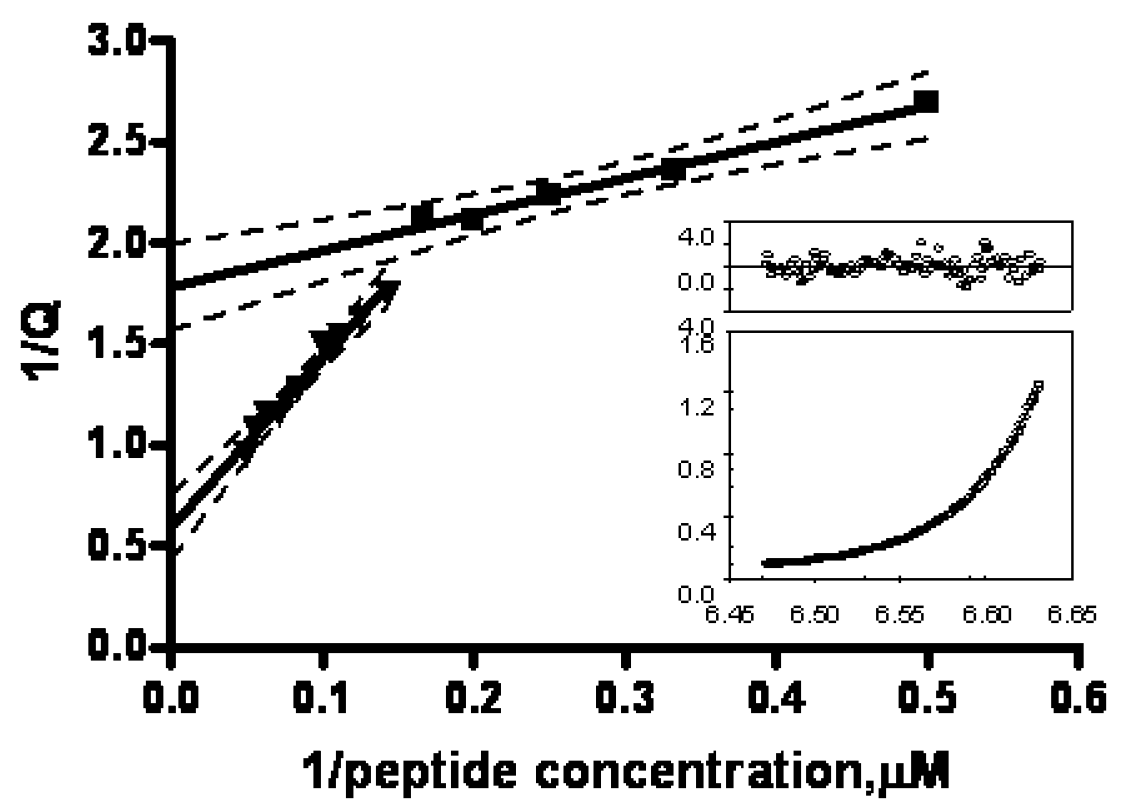

Fig. 2. Peptide NHR2 self association by the ANS test. The break point, at $\sim 4 \mu \mathrm{M}$, indicates the onset of oligomerisation. Inset: analytical equilibrium ultracentrifugation. Radial distribution of an NHR2 solution, fitted to a monomer - tetramer equilibrium with $K_{\mathrm{d}}=190 \mu \mathrm{M}^{-3}$. 


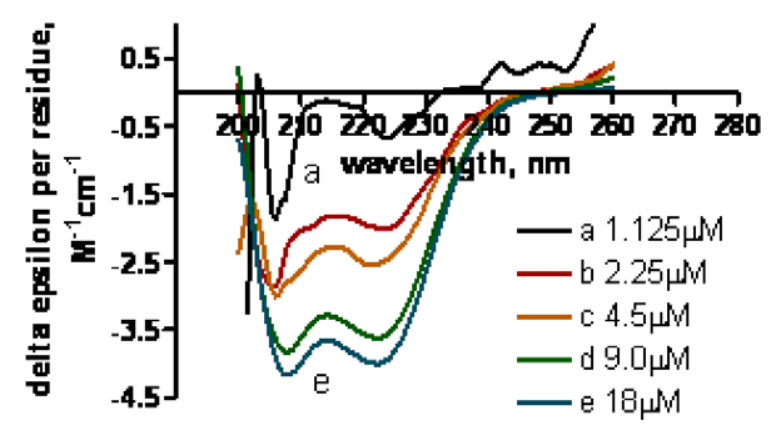

(a)

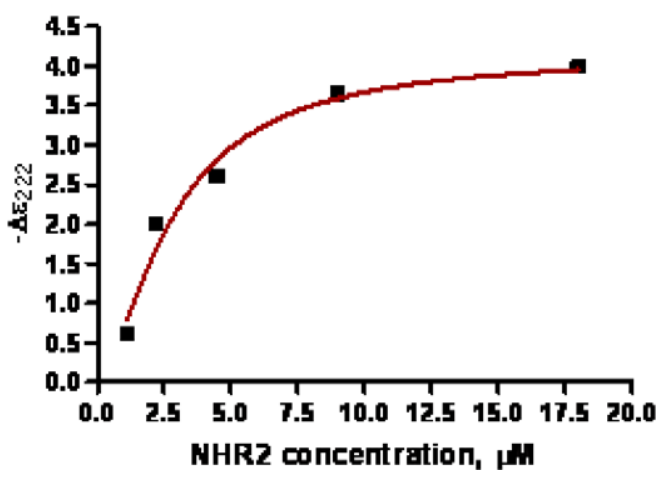

(b)

Fig. 3. Circular dichroism spectra of successive dilutions of peptide NHR2 (a). Plot of the molar differential absorptivity per residue at $222 \mathrm{~nm}$ as a function of NHR2 concentration (b). The titration midpoint is at $2.8 \mu \mathrm{M}$.

fluorescence enhancement passes from 0.56 to 1.78 when the peptide concentrations are situated above the breakpoint, i.e., the fluorescence exaltation increases from 56 to $178 \%$, which is consistent with peptide oligomerisation.

The CD titration of NHR2 with itself (Fig. 3) confirms the ANS result and elucidates the nature of the oligomerisation process. The CD titration midpoint is at $2.8 \mu \mathrm{M}$. It is within the same range as the breakpoint obtained previously with ANS, at $4.2 \mu \mathrm{M}$. The difficulty of obtaining more accurate data at these low concentrations prevented a full treatment of the binding curve. Above the titration midpoint the peptide displays obvious alpha helical features (negative peaks at 208 and $222 \mathrm{~nm}$ ). These features become less pronounced upon diluting the sample. The monomer seems to be unstructured at the lowest concentrations. It is thus apparent that the adoption of the secondary structure goes in parallel with the establishing of quaternary interactions, i.e., the onset of oligomerisation grants the seeding for helix formation. We have extensively discussed such situations [4,5,8]. In practical terms, it also emerges that, in order to avoid interference from the association process of peptide NHR2, one should work below $2.8 \mu \mathrm{M}$.

The distribution of the hydrophobic residues of peptide NHR2, creating an apolar spine (Fig. 4(a)), as well as the electrostatic potential at the solvent-accessible surface of the helix, positive at the C-end and negative at the opposite one (Fig. 4(b)), are both compatible with a coiled-coil, two-by-two antiparallel arrangement of the helices, as a first step in the oligomerisation leading to tetramer formation.

\subsection{Interaction of peptide TAFH with peptides NHR2 and AD1}

\subsubsection{Intrinsic fluorescence titrations}

Peptide NHR2 contains an LxxLL motif that resembles the one present in the AD1 domain of E-protein, the "partner" of the TAFH segment of AML1-ETO (Table 1 and Fig. 1(a)). It was therefore interesting to check whether a TAFH-NHR2 interaction did exist (Fig. 1(b)). It will be recalled that peptide NHR2 is the only species in this experiment to contain a tryptophane residue.

The plot of the fluorescence intensities of NHR2, at its maximum emission wavelength, against TAFH concentration suggests that the interaction with TAFH is strong (Fig. 5). With peptide NHR2 maintained at $0.5 \mu \mathrm{M}$, the two straight-line portions of the graph meet at $0.5 \mu \mathrm{M}$ TAFH concentration, suggesting a 1:1 stoichiometry for the TAFH-NHR2 complex. The result is an interesting one. Indeed, in addition to (and different from) the self association of NHR2, which leads to tetramer formation (see above; 

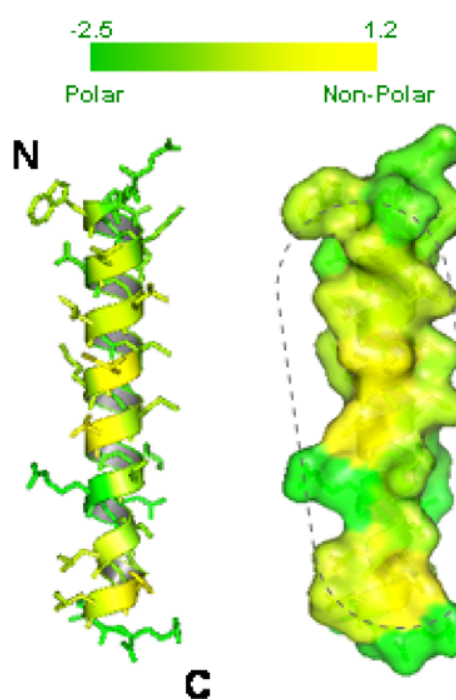

C

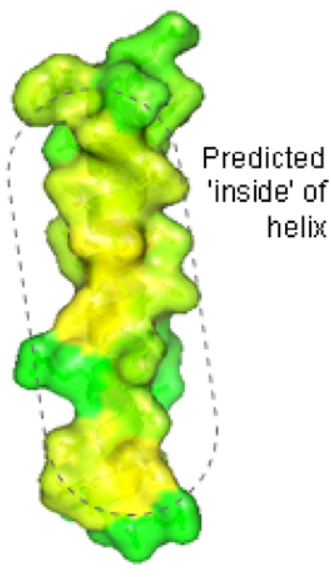

(a)

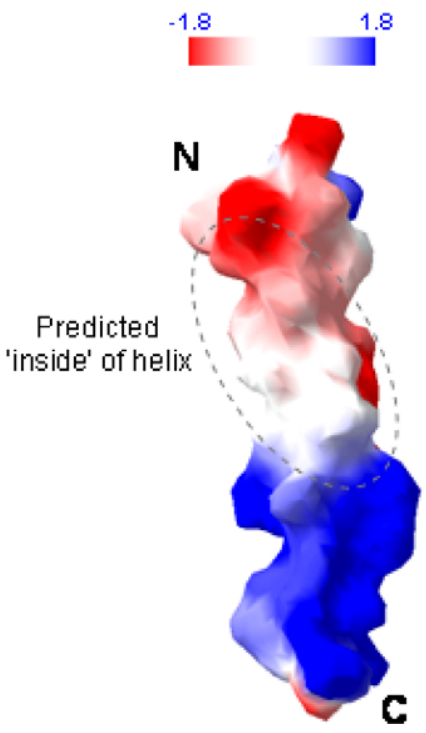

(b)

Fig. 4. Physical properties of peptide NHR2: hydrophobicity (Eisenberg scale, a); and surface potential, with the N-terminal being positive and the $\mathrm{C}$-terminal negative (b). The residues were attached to the skeleton of a standard $\alpha$-helix. Representation is by PyMol.

\section{Titration of NHR2 peptide at $0.5 \mu M$ by TAFH Determination of the stoichiom etry}

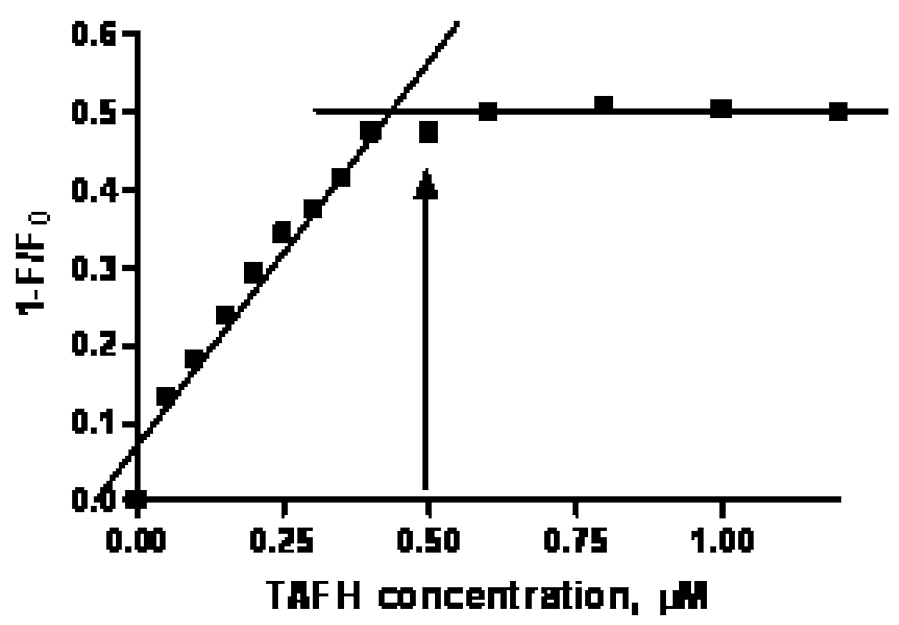

Fig. 5. Stoichiometry of the NHR2-TAFH interaction, by intrinsic fluorescence: titration of peptide NHR2, at $0.5 \mu$ M, by TAFH shows a 1:1 stoichiometry.

recall that a tetramer structure of the NHR2 domain is essential for the AML1-ETO activity [3]), the association of NHR2 with TAFH only goes up to the hetero dimer level.

The corollary is that the TAFH domain would probably recognize at least another partner bearing the LxxLL motif and, before binding to AD1, the first such interaction could be intramolecular, within the AML1-ETO protein itself (Fig. 1(b)). 


\subsubsection{Determining the binding constants for the interaction of TAFH with peptides NHR 2 and AD1}

The binding constants for the interaction of TAFH with peptides NHR2 and AD1 were determined by adapting a program based on the Newton-Raphson method, originally developed by Linse et al. for the monitoring of calcium binding to proteins in the presence of a calcium-sensitive dye [1,2].

At first, the titration of peptide NHR2 with peptide TAFH in the absence of any competitor yielded the value $K_{\mathrm{d}}=1.28 \mathrm{nM}$ for the "intramolecular" TAFH-NHR2 interaction (Fig. 6(a)). This value was used in the analysis of a second series of titrations, where peptide AD1 was present as a "competitor" for the NHR2 to TAFH interaction. In this way, one obtained the value $K_{\mathrm{d}}=28 \mathrm{nM}$ for the binding of TAFH to AD1. This "intermolecular" interaction is fairly strong as well. If it appears less than the intramolecular one, this is certainly due to the smaller size of the AD1 peptide (peptide NHR2 has 29 residues, as opposed to 17 residues in peptide AD1 - see Table 1).

In the same way, one titrated peptide NHR2 with peptide AD1 and obtained a value of $K_{\mathrm{d}}=240 \mathrm{nM}$ for their mutual interaction. Since peptide NHR2 is known to auto associate, and since both peptides NHR2 and AD1 contain LxxLL motifs, it is no surprise that the two peptides bind to each other. Whereas

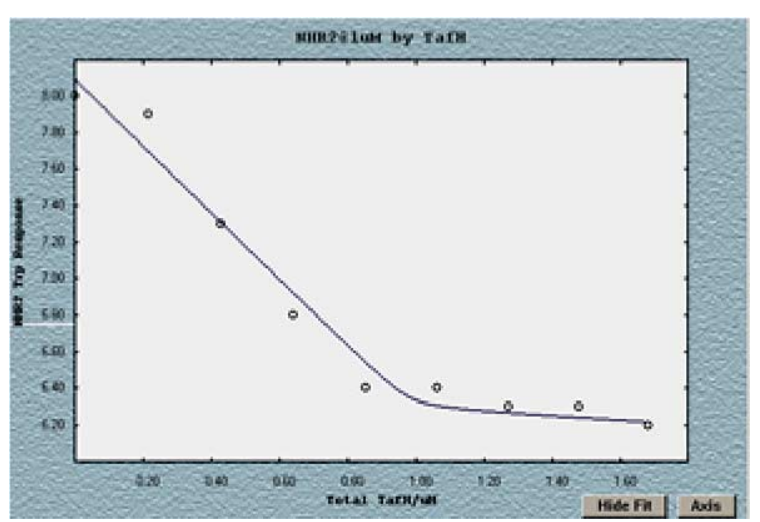

(a)

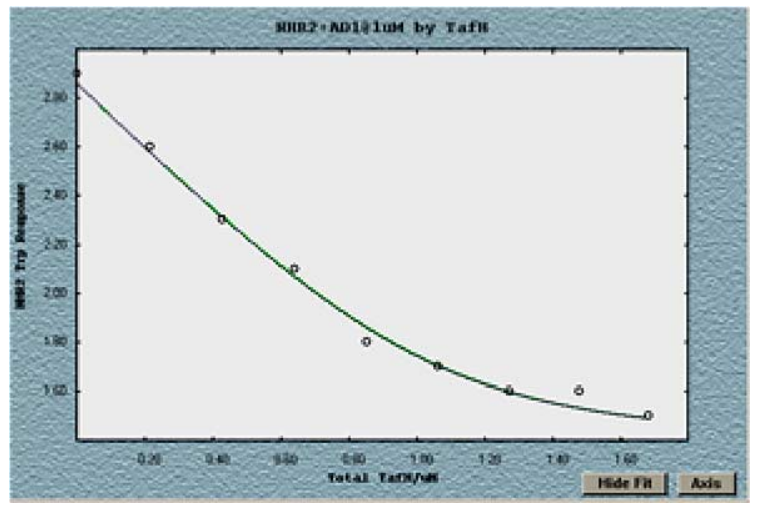

(b)

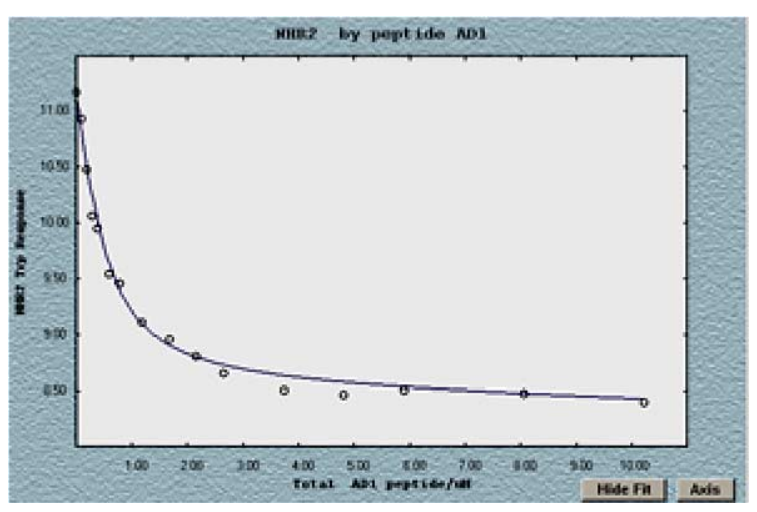

(c)

Fig. 6. Binding constants. Competitive assays, analyzed by the Newton-Raphson method, using the Caligator software $[7,8]$. The Trp fluorescence from NHR2 was monitored as a function of TAFH concentration, in the absence of any competitor, for the TAFH-NHR2 interaction $\left(K_{\mathrm{d}}=1.28 \mathrm{nM}\right.$, panel a); as a function of TAFH in the presence of AD1 as competitor, for the binding of TAFH to AD1 ( $K_{\mathrm{d}}=28 \mathrm{nM}$, panel b); and as a function of AD1 alone, for the mutual interaction of AD1 and NHR2 $\left(K_{\mathrm{d}}=240 \mathrm{nM}\right.$, panel c). The absorbance values in the plots involving TAFH have been corrected for Tyr contribution by subtracting TAFH intensities, obtained separately. 
the first two pieces of data indicate that the NHR2 with AD1 peptides interact with TAFH very strongly, the latter tells that a less intense mutual interaction between the two partners bearing LxxLL motifs does exist, possibly as an artefact.

\section{Conclusions}

We have described the nature of the oligomerisation process involving the NHR2 domain of AML1ETO. We hypothesized an antiparallel encounter of two amphipathic molecules, mutually reinforcing their helical structures while adopting a coiled-coil conformation, as a prerequisite to tetramer formation. On the other hand, we have shown that the TAFH domain could recognize an additional bearing the LxxLL motif and, before binding to AD1, the first such interaction is likely to be intramolecular, with the NHR2 domain of the AML1-ETO protein itself. Furthermore, a weak interaction of NHR2 with AD1 was also revealed.

We have obtained dissociation constants for the self association of peptide NHR2 $\left(K_{\mathrm{d}}=2.8-4 \mu \mathrm{M}\right.$, depending on the method used), for the binding of peptide NHR2 to TAFH ( $K_{\mathrm{d}}=1.28 \mathrm{nM}$, with 1:1 stoichiometry) and to $\mathrm{AD} 1\left(K_{\mathrm{d}}=240 \mathrm{nM}\right)$ and for the TAFH-AD1 interaction $\left(K_{\mathrm{d}}=28 \mathrm{nM}\right)$.

Of the intramolecular interaction between the NHR2 and TAFH domains of AML1-ETO, signalled as a possibility, one may expect a modulator role. It could be involved in the control of the oligomerisation of AML1-ETO via the NHR2 domain and/or the interaction of the TAFH domain with the AD1 domain of E-protein. The elucidation of the structure adopted by peptide AD1 upon binding to TAFH will facilitate the modelling of the local structure of the TAFH-AD1 complex, unclear at the time of writing [7].

The multiple interactions involving the LxxLL motif, revealed by this work, raise the possibility of using modified LxxLL-containing peptides as targeted modulators of the intramolecular TAFH-NHR2 interaction, of the NHR2-based oligomerisation of AML1-ETO and of the AML1-ETO-E-protein complex.

\section{Acknowledgements}

The author acknowledges with gratitude the collaboration with, and provision of excellent facilities by M. Ikura and M. Plevin, from the Division of Signaling Biology, Ontario Cancer Institute, University Health Network, Toronto, ON, Canada. He is thankful to Sara Linse for the Caligator program [1,2]. The hospitality of Jasco, Easton, MD is gratefully recognized.

\section{References}

[1] I. Andre and S. Linse, Anal. Biochem. 305 (2002), 195-205.

[2] S. Linse, Methods Mol. Biol. 173 (2002), 15-24.

[3] Y. Liu, M.D. Cheney, J.J. Gaudet, M. Chruszcz, S.M. Lukasik, D. Sugiyama, J. Lary, J. Cole, Z. Dauter, W. Minor, N.A. Speck and J.H. Bushweller, Cancer Cell 9 (2006), 249-260.

[4] R.G. Maroun, D. Krebs, A.S. El, A. Deroussent, E. Lescot, F. Troalen, H. Porumb, M.E. Goldberg and S. Fermandjian, J. Biol. Chem. 274 (1999), 34174-34185.

[5] R.G. Maroun, D. Krebs, M. Roshani, H. Porumb, C. Auclair, F. Troalen and S. Fermandjian, Eur. J. Biochem. 260 (1999), $145-155$.

[6] M.J. Plevin, M.M. Mills and M. Ikura, Trends Biochem. Sci. 30 (2005), 66-69. 
[7] M.J. Plevin, J. Zhang, C. Guo, R.G. Roeder and M. Ikura, Proc. Natl. Acad. Sci. USA 103 (2006), 10242-10247.

[8] H. Porumb, L. Zargarian, H. Merad, R. Maroun, O. Mauffret, F. Troalen and S. Fermandjian, Biochim. Biophys. Acta 1699 (2004), 77-86.

[9] C. Schessl, V.P. Rawat, M. Cusan, A. Deshpande, T.M. Kohl, P.M. Rosten, K. Spiekermann, R.K. Humphries, S. Schnittger, W. Kern, W. Hiddemann, L. Quintanilla-Martinez, S.K. Bohlander, M. Feuring-Buske and C. Buske, J. Clin. Invest. 115 (2005), 2159-2168.

[10] Y. Yuan, L. Zhou, T. Miyamoto, H. Iwasaki, N. Harakawa, C.J. Hetherington, S.A. Burel, E. Lagasse, I.L. Weissman, K. Akashi and D.E. Zhang, Proc. Natl. Acad. Sci. USA 98 (2001), 10398-10403. 


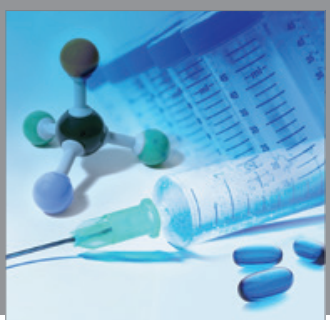

International Journal of

Medicinal Chemistry

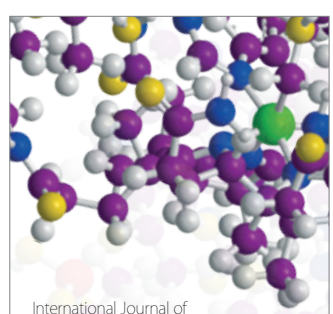

Carbohydrate Chemistry

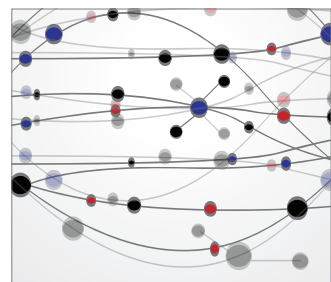

The Scientific World Journal
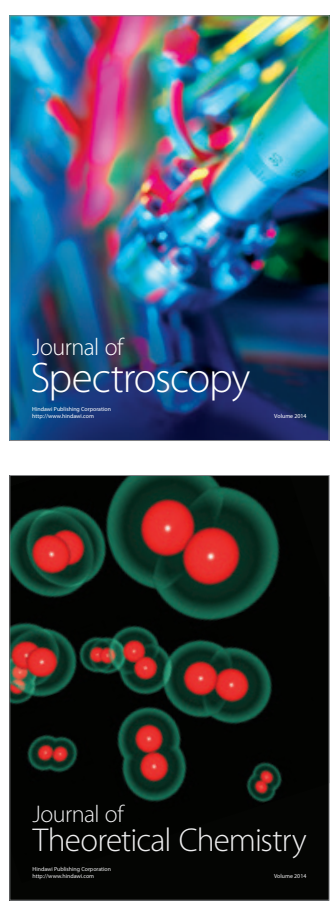
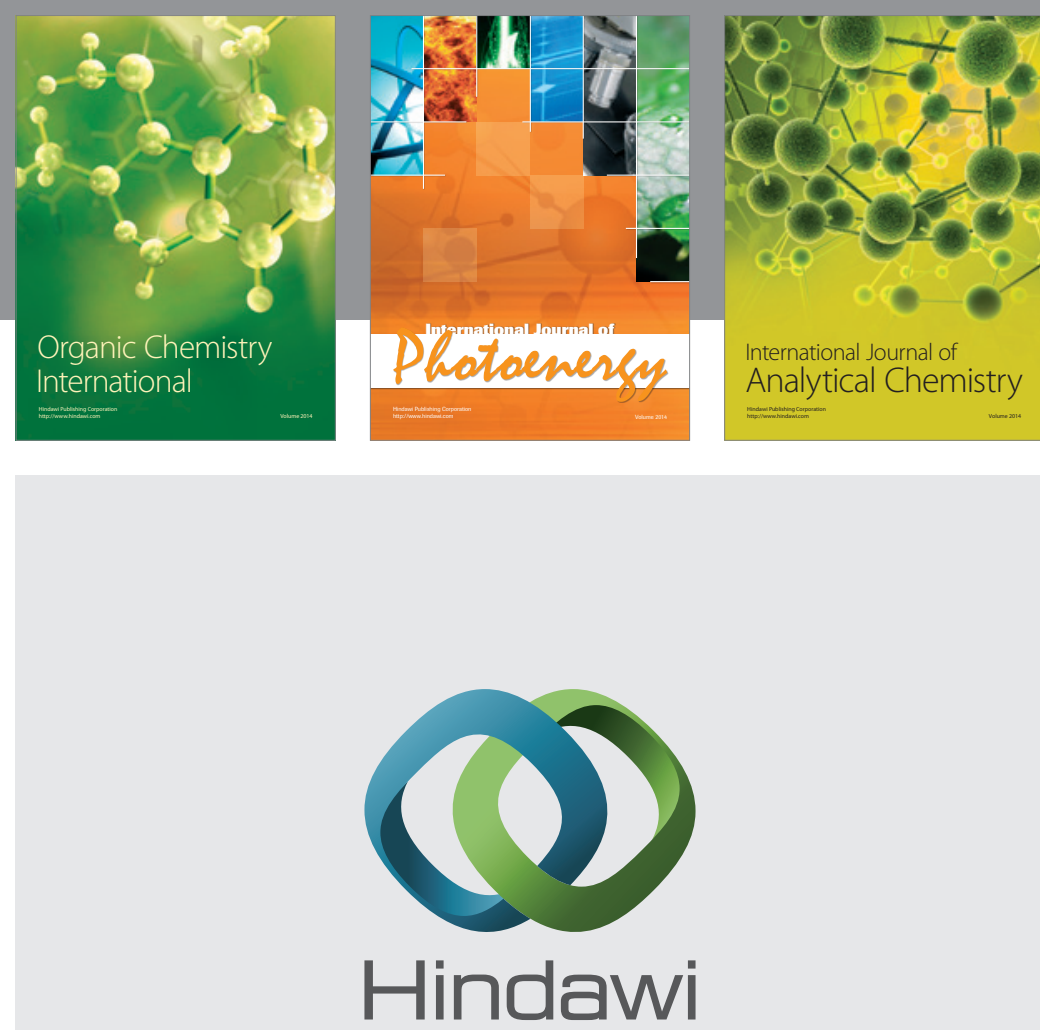

Submit your manuscripts at

http://www.hindawi.com
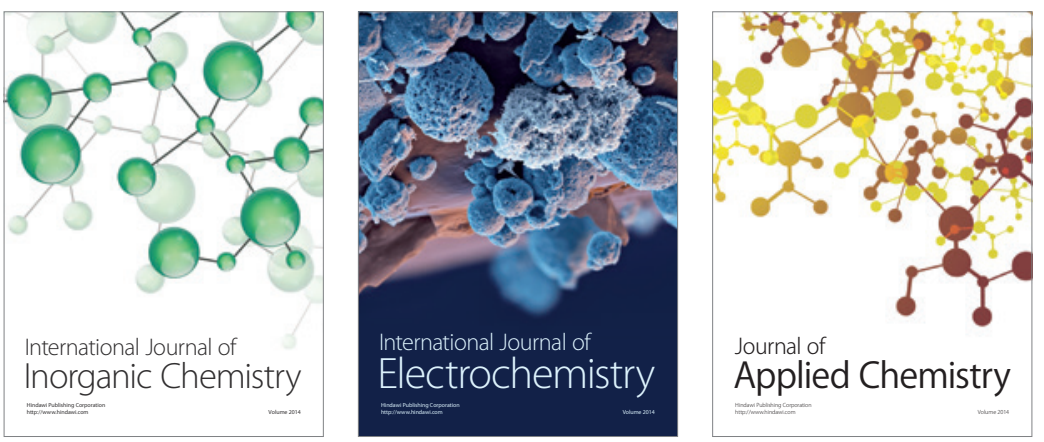

Journal of

Applied Chemistry
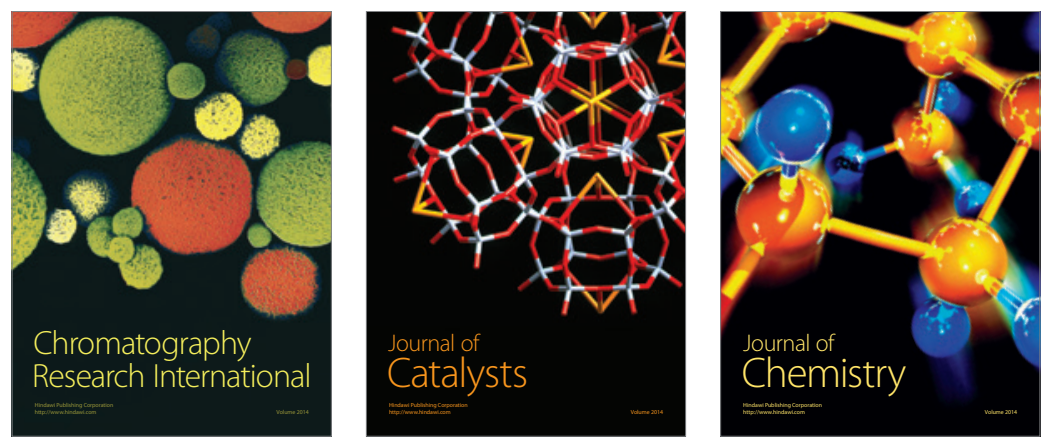
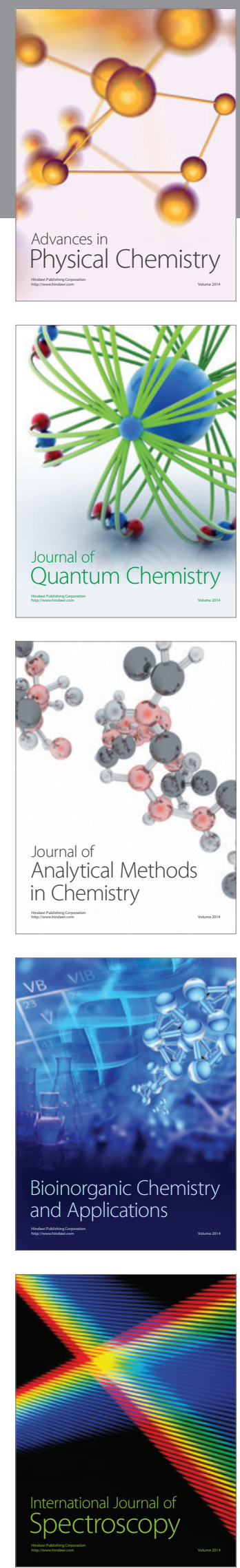